\title{
Colgajo de reposición apical
}

\section{Apically positioned flap}

\author{
PÉREZ-SALCEDO L* \\ BASCONES MARTÍNEZ A**
}

Pérez-Salcedo L, Bascones Martínez A. Colgajo de reposición apical. Av Periodon Implantol. 201 1; 23, 2: 91-96.

\begin{abstract}
RESUMEN
Se describe la técnica del colgajo de reposición apical desde el primer autor, Nabers en 1954, así como las modificaciones realizadas por Ariaudo y Tyrrel en 1957 y Friedman en 1962. También se presenta un caso clínico donde se describen los diferentes pasos de la cirugía de reposición apical. Por último, se hace una revisión evaluando la eficacia del colgajo de reposición apical en el tratamiento de la periodontitis.
\end{abstract}

PALABRAS CLAVE: Colgajo de reposición apical.

\section{SUIMIMARY}

This paper describes the technique apically positioned flap since the first author Nabers in 1954 and also the modifications performed by Ariaudo and Tyrrell in 1957 and Friedman in 1962. Also a clinical case is presented where it can be observed the different steps of the apically positioned flap in clinical pictures. Afterwards some papers in which the efficacy of the apically positioned flap in the treatment of periodontitis are evaluated and described.

KEYWORDS: Apically positioned flap.

Fecha de recepción: 9 de junio de 2009.

Fecha de aceptación: 22 de junio de 2009.

\section{INTRODUCCIÓN}

El colgajo de reposición apical fue diseñado por Nabers en 1954 definiéndolo como Reposición Apical de la encía adherida (1). Este autor diseñó el colgajo para mantener la encía insertada debido a sus características histológicas y funcionales.

El autor describe diferentes características de la mucosa alveolar y encía queratinizada. (2) En cuanto a la mucosa alveolar refiere la gran cantidad de fibras elásticas que contiene y su no queratinización en cuanto a su función se afirma que es el recubrimiento de la cavidad oral. En cuanto a la encía adherida describe su composición formada por tejido conectivo denso y caracterizándose por estar firmemente adherida al hueso y ser un epitelio estratificado queratinizado.
El diseño quirúrgico se realiza en los casos en los que la base de la bolsa esté localizada en la mucosa alveolar y consiste en la realización de una descarga en mesial, elevar el colgajo para desbridar, eliminar el tejido de granulación; a su vez se realiza un rodete de no más de $2 \mathrm{~mm}$ y por último se reposiciona apicalmente.

Ariaudo y Tyrrel en 1957 (3) modifican la técnica dando una descripción más detallada. En este artículo se presenta esta cirugía para cumplir varios objetivos: aumentar la dimensión oclusoapical de la encía adherida, eliminar la bolsa periodontal y lograr la reinserción de la bolsa infraósea (3).

Para ello describe la técnica realizando una pequeña modificación con respecto a la descrita por Nabers

* Máster de Periodoncia e Implantología UCM.

** Catedrático de Medicina Bucal y Periodoncia e Implantes UCM. 
1954 (1), realizando 2 incisiones en mesial y en distal del colgajo (3).

- Los irritantes como cálculo, tejido de granulación deben eliminarse y las raíces alisadas. Se debe realizar un rodete de no más de $2 \mathrm{~mm}$ del epitelio crevicular con unas tijeras curvas.

- El hueso de la pared ósea debe ser o no eliminado según el criterio del operador/a.

- El colgajo debe ser reposicionado apical a la base de la bolsa preexistente, dejando hueso expuesto.

- El colgajo se mantiene en la posición deseada mediante una ligera presión hasta que cesa el sangrado y el coágulo de fibrina mantiene el colgajo en la posición deseada.

- Se coloca cemento quirúrgico con hidrocoloide de tetraciclina.

- Se administran antibióticos a criterio del operador y se cita cada 5 días. Según avanza la cicatrización el tejido de granulación cubre el hueso expuesto y este tejido se convierte en encía adherida de manera que la antigua encía adherida junto con la nueva formada doblan la dimensión oclusoapical de este tejido.

El autor enumera una serie de ventajas de esta técnica con respecto a la de eliminación de bolsas utilizada en los años 50 que era la gingivectomía que son la mejor visibilidad de los irritantes locales como el cálculo, tejido de granulación, hueso alveolar, bolsas intraóseas...

También se enumera una serie de desventajas de esta técnica:

- Más dolor para el paciente en relación a la gingivectomía.

- Mayor número de citas que deben continuar hasta la semana 3 ó 4 para mantener el cemento quirúrgico en la herida hasta que la epitelización es completa.

- En algunos casos el colgajo se desplaza apical más de lo deseado.

- La osteoplastia está indicada dependiendo de la morfología ósea.

- En caso de que se necesite una gingivectomía en lingual se debe hacer en la misma cita, a veces se necesita una gingivoplastia posterior debido al mal contorno de los tejidos gingivales.

En 1962 Friedman (3) modifica la técnica e incluso el nombre de la técnica ya que al reposicionar todo el complejo de encía y mucosa alveolar y no sólo la encía adherida sustituye el nombre de colgajo reposición de encía adherida por colgajo de reposición apical.

Los objetivos son: la eliminación de la bolsa periodontal que consigue que no se retraigan los tejidos al traccionar del frenillo y obtiene suficiente profundidad de vestíbulo.

En primer lugar se recomienda el raspado y alisado radicular (RAR) y el ajuste oclusal. Posteriormente y en caso de ser necesaria la cirugía, se realiza una incisión horizontal a bisel interno (aproximadamente $25^{\circ}$ ) a una distancia del margen gingival que depende de la profundidad de sondaje, la cantidad de encía y el grosor de esta. Se realizan 2 incisiones liberadoras extendiéndose más allá de la línea mucogingival, elevando posteriormente el colgajo y eliminando el rodete de tejido, se raspan y alisan las raíces y se realiza un remodelado óseo con rotatorios $(4,5,6)$ para recuperar la forma de un periodonto normal. Para finalizar se reposiciona apicalmente el colgajo y se sutura de manera que queda hueso expuesto sólo en interproximal, en estas zonas se coloca cemento quirúrgico (Fig. 1).

Las ventajas de la cirugía de reposición apical son la cicatrización por $1^{a}$ intención, más rápida y menos dolorosa. Se obtiene mayor recubrimiento de hueso por tejido de manera que se evitan los secuestros óseos, se consigue mejor control de la cantidad de encía postoperatoria, el colgajo queda confinado a los dientes problema y por último se profundiza el vestíbulo.

En cuanto a las desventajas son la mayor dificultad de la técnica debido a la importancia de suturar firmemente y al adelgazamiento del colgajo, en caso de poca encía prequirúrgica el colgajo es difícil de manejar (7). Incluso el autor recomienda usar otras técnicas en casos de $2^{\circ}$ y $3^{\text {er }}$ molares inferiores con vestíbulos poco profundos (Fig. 2).

Un concepto importante que describe este autor en su artículo es el colgajo biselado palatino (8) ya que en palatino no hay línea mucogingival el colgajo no se puede reposicionar, el autor propone elevar un colgajo similar a la descripción de la técnica en vestibular, realizando RAR y remodelado óseo y posteriormente realizar el festoneado del colgajo de manera que se observa cuanto tejido es necesario eliminar para cubrir el hueso de manera adecuada. Por último se sutura con puntos simples en interproximal (Fig. 3). 




Fig. 1.

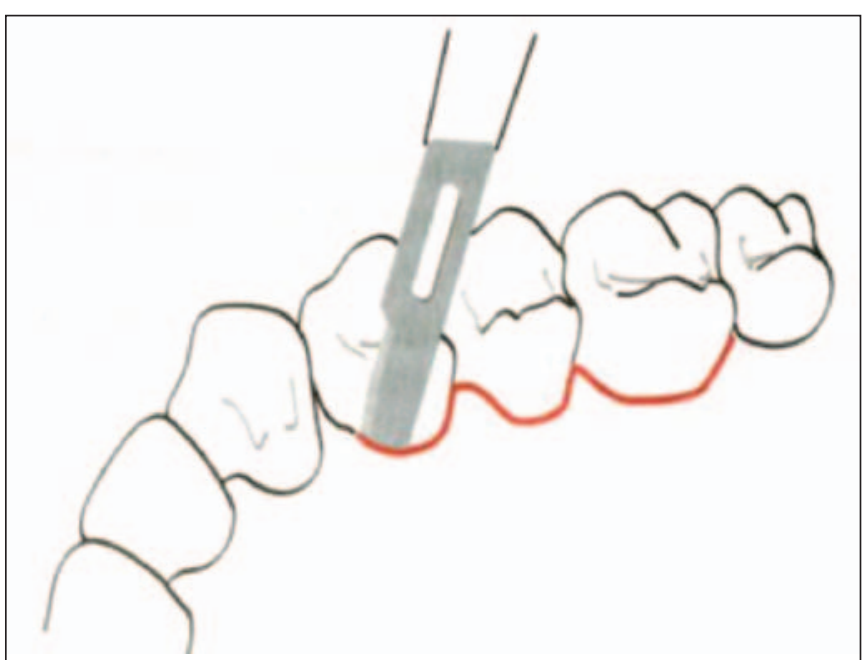

Fig. 2.

Se presentan fotos de un caso clínico en el que se realizó una cirugía de reposición apical en el sexto sextante. Es importante destacar que a los pacientes que padecen periodontitis se les debe realizar en primer lugar el tratamiento periodontal básico que consiste en Instrucciones de higiene oral, RAR, y ajuste oclusal en caso de ser necesario y por supuesto el tratamiento restaurador que requiera cada paciente. Una vez realizado este tratamiento se debe reevaluar al paciente en un mínimo de tiempo de 1 mes y en caso de persistencia de bolsas profundas se realizará el tratamiento quirúrgico necesario que en este caso es una cirugía de reposición apical .

Se realiza una incisión intrasulcular en vestibular y con un rodete de aproximadamente $2 \mathrm{~mm}$ en lingual. Debido a la falta de encía queratinizada no se procede a realizar un rodete más extenso (Fig. 4).

Una vez levantado el colgajo, se elimina el tejido de granulación y se raspan las raíces (Fig. 5).

En la figura 6 se observa la diferencia en la anatomía ósea antes de realizar la cirugía ósea y después. Se realiza osteoplastia de manera que los colgajos apongan mejor sobre el hueso y también ostectomía para disminuir el defecto que presenta en 47.

Se realiza una sutura continua dentoanclada y puntos simples en la cresta edéntula (Fig. 7).

Kaldahl en 1996 (9) realiza un estudio para evaluar a largo plazo la respuesta al tratamiento de 4 modali- 


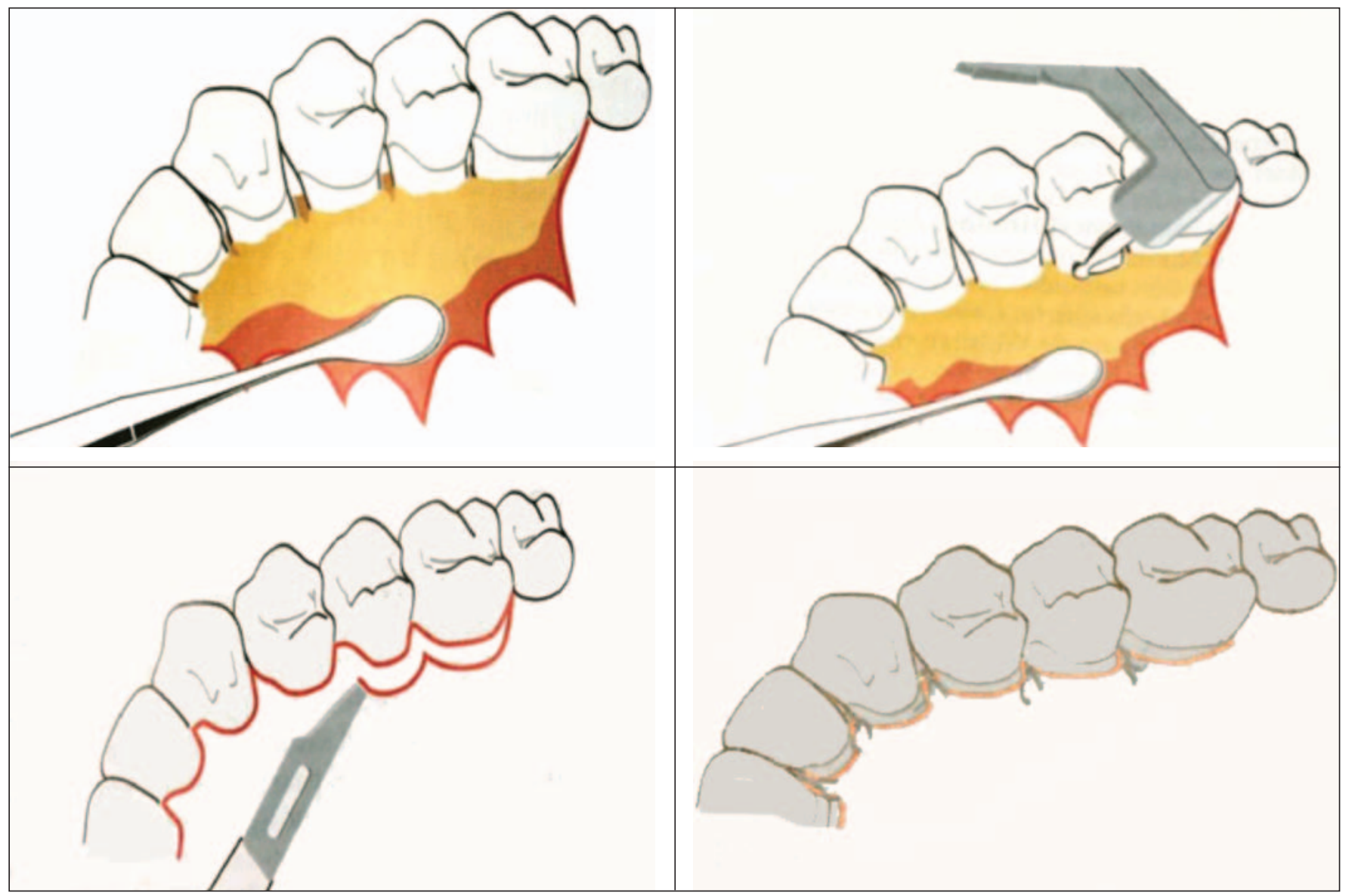

Fig. 3.

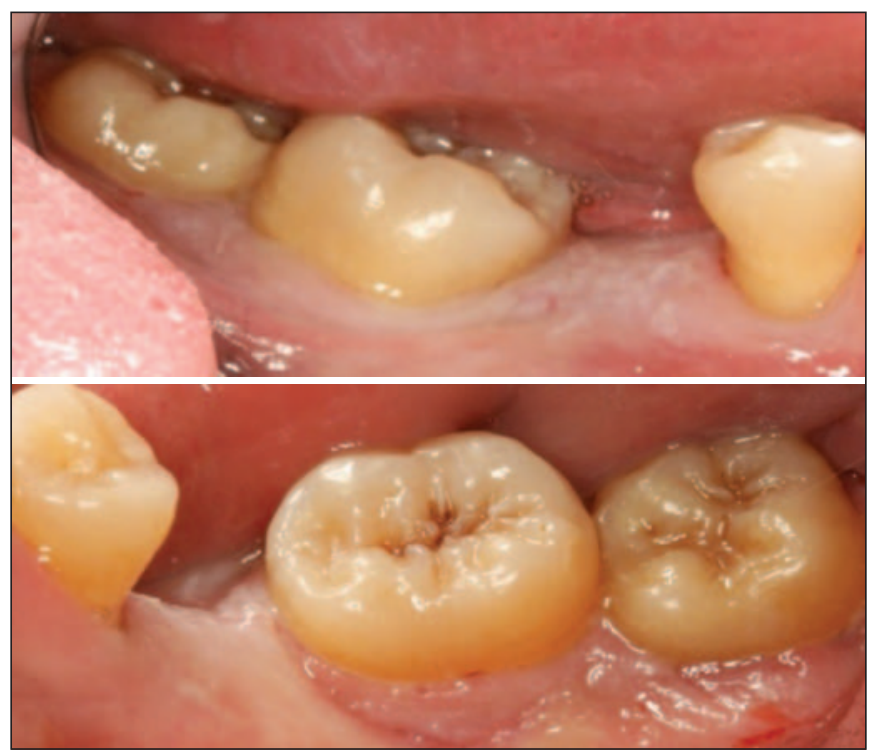

Fig. 4. dades terapéuticas, para ello utiliza 82 pacientes con periodontitis de moderada a avanzada y en cada cuadrante realiza un tratamiento: raspado coronal, raspado y alisado radicular (RAR), colgajo de Widman modificado (CWM) y colgajo de reposición apical (CRA). El diseño experimental consiste en 3 fases de tratamiento:

1. Fase 1: Los pacientes reciben instrucciones de higiene oral y se realiza el raspado coronal en los dientes seleccionados para ello y RAR para el resto de modalidades terapéuticas.

2. Fase 2: Se realizan las cirugías, en un cuadrante se realiza CRA y en otro CWM. En el caso de CRA se eleva el colgajo y se realiza ostectomía hasta obtener arquitectura ósea positiva.

Las cirugías se realizaron en localizaciones con profundidades de sondaje mayores o iguales a $5 \mathrm{~mm}$ y nunca en sectores anteriores. 


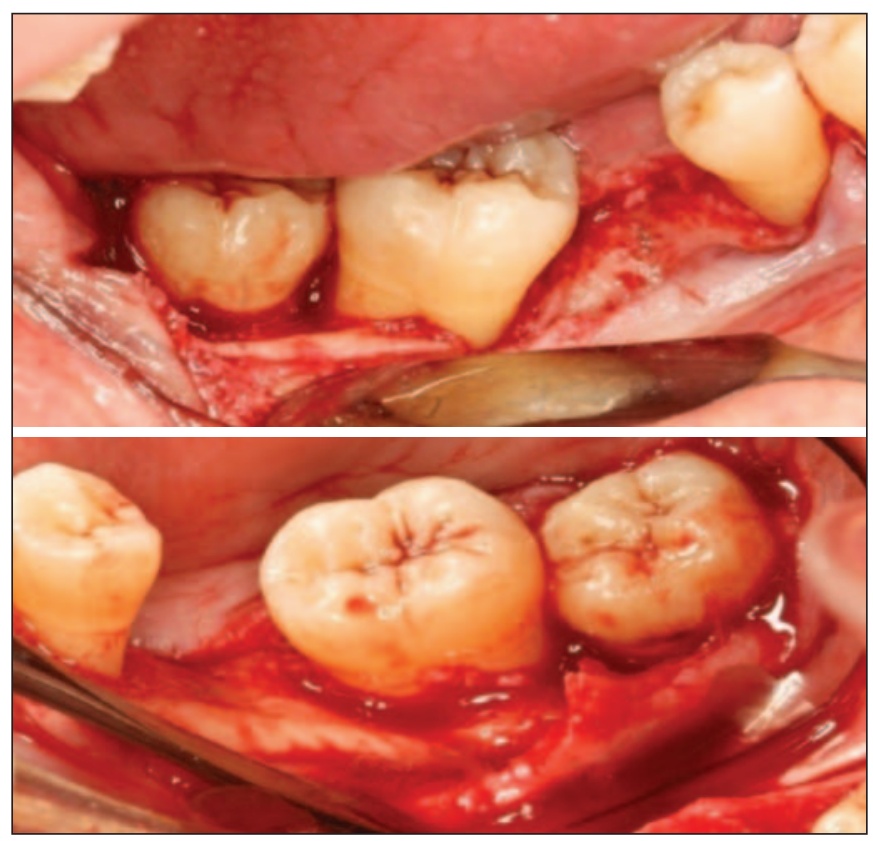

Fig.5.

3. Fase 3: La última fase de tratamiento consiste en el mantenimiento periodontal que en este caso se realiza cada 3 meses y consiste en instrucciones de higiene oral, raspado coronal y RAR en caso de ser necesario pero no en los dientes seleccionados sólo para el raspado coronal.

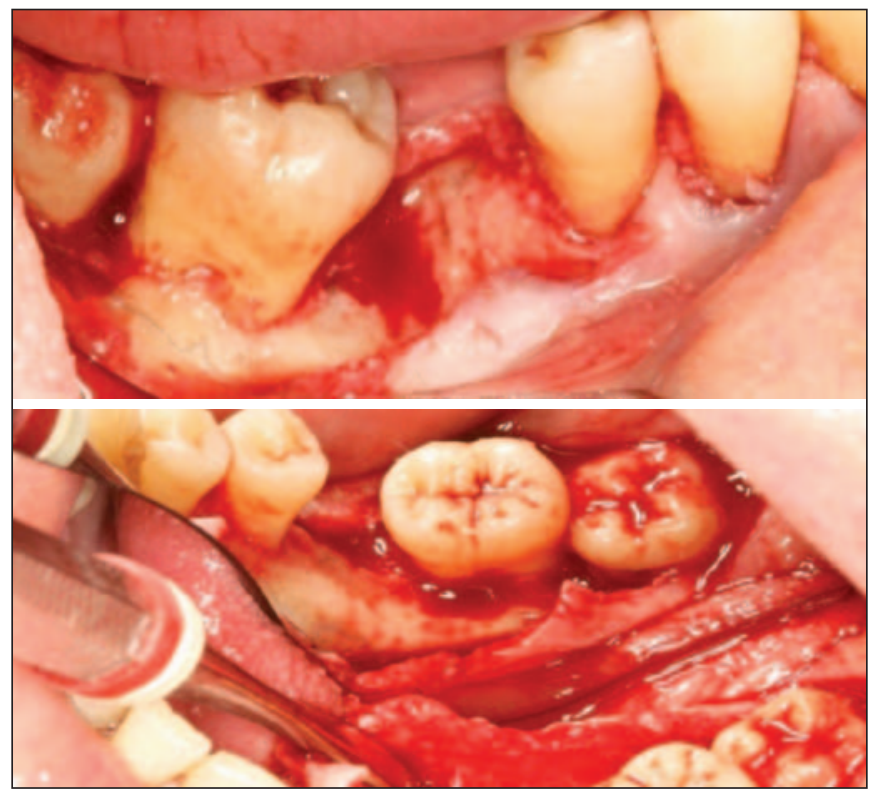

Fig. 6.
Las variables evaluadas fueron el índice de placa, supuración, sangrado al sondaje, profundidad de sondaje, recesión y nivel de inserción.

En cuanto a los resultados en la profundidad de sondaje la mayor reducción de bolsa la obtiene el CRA y a su vez también obtiene la mayor estabilidad a largo plazo de los resultados. Esta reducción es incluso mayor en bolsas de profundidades mayores de $7 \mathrm{~mm}$.

En cuanto a la ganancia del nivel de inserción en bolsas de 5-6 mm se observa mayor ganancia de inserción con el RAR y entre el CWM y el CRA no hay diferencias. En cambio en bolsas mayores de $7 \mathrm{~mm}$ se obtiene la misma ganancia del nivel de inserción tanto con RAR, CWM y CRA.

Los autores concluyen que tras realizar este ensayo clínico a 7 años se demuestra que todos los tratamientos mejoran las variables clínicas.

En cambio también existen artículos en los que los autores afirman que no existen diferencias en cuanto a las variables clínicas con las diferentes modalidades de tratamiento. Uno de ellos es Becker \& Becker del 2001 (10), que publican un ensayo clínico aleatorizado con el objetivo de evaluar los resultados clínicos de tres modalidades de tratamiento con un periodo de seguimiento de 5 años. Para ello utilizan 16 pacientes con periodontitis de moderada a avanzada a los

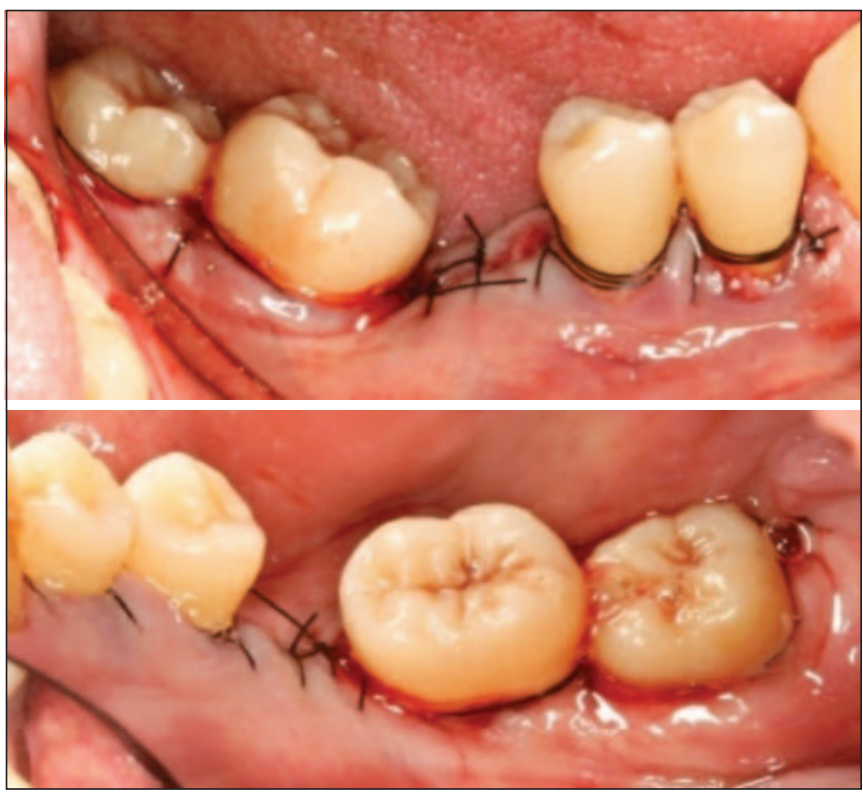

Fig. 7. 
que realizan RAR y posteriormente seleccionan el tipo de tratamiento bien CRA o bien CWM. Posteriormente los pacientes se incluyeron en un programa de mantenimiento trimestral.

Las variables evaluadas son el índice de placa, índice gingival, profundidad de sondaje, nivel de inserción y recesión.

En cuanto a los resultados no existen diferencias entre las 3 modalidades de tratamiento ni en el índice de placa ni en el índice gingival. Tampoco existen diferencias en la reducción de profundidad de bolsa ni en la ganancia del nivel de inserción.

Los autores concluyen que se pueden obtener buenos resultados y mantenerlos durante 5 años con las 3 modalidades de terapia evaluadas.

\section{CONCLUSIONES}

1. La cirugía de reposición apical implica un diseño con rodete, despegamiento del colgajo hasta más allá de la línea mucogingival, cirugía ósea y reposición apical del colgajo, aunque en la actualidad en una misma cirugía se realizaran rodetes donde sea necesario y exista suficiente encía y la cirugía ósea no debe comprometer a los dientes adyacentes.

2. La cirugía de reposición apical ha demostrado ser un procedimiento quirúrgico efectivo en el tratamiento de la periodontitis.

3. Este tipo de cirugía no se recomienda en los sectores anteriores que tengan compromiso estético.

4. Aunque se consigan todos los objetivos con el tratamiento periodontal para que éstos se mantengan a largo plazo es necesario el mantenimiento periodontal que debe consistir en un examen periodontal exhaustivo que incluya un periodontograma completo con índice de placa, sangrado al sondaje, profundidad de bolsa, recesiones, furcas y movilidad. De manera que se pueda comparar con las mediciones tomadas tras el tratamiento periodontal. Se realizará en estas sesiones de mantenimiento una tartrectomía supragingival y raspado y alisado radicular de las bolsas profundas e instrucciones de higiene oral.
5. Se deben adecuar los intervalos de mantenimiento a las necesidades de cada paciente.

\section{BIBLIOGRAFÍA}

1. Nabers C. Repositioning the attached gingiva. Journal of periodontology. 1954;25:38-9.

2. Orban B. Oral Histology and Embriology. C.V Mosby Company 1947, pp.269.

3. Ariaudo AA, Tyrrell HA. Repositioning and increasing the zone of attached gingiva. Journal of periodontology. 1957 April;28:106-10.

3. Friedman N. The apically repositioned flap. Journal of periodontology. 1962;33:328-40.

4. Schluger S. Osseous Resection. A basic principle in periodontal surgery? Oral Surg., Oral Med. And Oral Path. 2:316 (March) 1949

5. Friedman N. Periodontal Osseous Surgery; Osteoplasty and Osteoctomy. J. Periodont. 26: 257 (October) 1957.

6. Prichard JF. Gingivoplasty, Gingivectomy, and Osseous Surgery. J. Periodont.32: 275 (October) 1961.

7. Ochsenbein, C. Newer concepts of mucogingival surgery. J.Periodont. 31:175(July) 1960.

8. Prichard JF. Peridontal Surgery. Pract Dent. Monographs (November) 1961.

9. Kaldahl et al. Long-term Evaluation of periodontal therapy: I. Response to 4 therapeutic modalities. J Periodontol 1996: 67:93-102.

10. Becker et al. A longitudinal study comparing scaling, osseous surgery and modified widman procedures. Results after one year.J Periodontol 1988:59(6)351-365.

\section{CORRESPONDENCIA}

\author{
L. Pérez-Salcedo \\ leireperezsalcedo@hotmail.com
}

